\title{
Tumores del Estroma Gastrointestinal (GIST): Características Clinico-Morfológicas y Perfil Inmunohistoquímico
}

\author{
Gastrointestinal Stromal Tumors (GIST): Clinical-morphological \\ Features and Immunohistochemical Profile
}

Oscar Tapia E. \& Juan Carlos Roa S.

TAPIA, E. O. \& ROA, S. J. C. Tumores del estroma gastrointestinal (GIST): características clinico-morfológicas y perfil inmunohistoquímico. Int. J. Morphol., 29(1):244-251, 2011.

RESUMEN: Los tumores del estroma gastrointestinal (GIST) son neoplasias de origen mesenquimático que representan aproximadamente el 0,3\% de todas las neoplasias del tubo digestivo, caracterizadas inmunohistoquímicamente por expresar CD117 en el 95\% de los casos y afectando más frecuentemente estómago e intestino delgado. El objetivo de este estudio fue describir aspectos clínicos, morfológicos y de inmunohistoquímica en pacientes con diagnóstico de GIST en la Unidad de Anatomía Patológica del Hospital Hernán Henríquez Aravena de Temuco, Chile. Estudio de cohorte retrospectiva. Se estudiaron 30 pacientes con diagnóstico de GIST intervenidos entre 1999 y 2010 en el Hospital Hernán Henríquez Aravena de Temuco. Las variables clínicas y morfológicas estudiadas fueron edad, género, localización y tamaño tumoral, tipo histológico, índice mitótico, compromiso de mucosa, grado de pleomorfismo nuclear y presencia de necrosis. El estudio inmunohistoquímico consideró c-KIT, CD34 y S-100. Se utilizaron estadísticas descriptivas y analíticas; aplicando chi-cuadrado de Pearson y exacto de Fisher para las variables categóricas; y, T-test para variables continuas. El promedio de edad fue 60 años (17-81 años), verificándose un 60\% de mujeres en el grupo estudiado. El 90\% correspondió a tumores de localización gastro-intestinal, representando estómago e intestino delgado el $80 \%$ de los casos. El tamaño tumoral promedio fue 75,9 mm. Correspondió a patrón fusocelular el 77\%, observándose necrosis en el 37\% de los casos. El 50\% presentó > 5 mitosis/50 CAM, verificándose compromiso de la mucosa en un $67 \%$. Según el grupo pronóstico se verificó $7 \%$ grupo 1, 23\% grupo 2, 20\% grupo 3, 0\% grupo $4,10 \%$ grupo 5 y $40 \%$ grupo 6 . El 100\% expresó positividad para c-KIT, 63\% para CD34 y 3\% para S-100. Los GIST afectan mayormente a pacientes entre la $4^{\circ}-6$ décadas de la vida con un leve predominio en mujeres, siendo el estómago e intestino delgado los órganos más frecuentemente afectados. El estudio inmunohistoquímico mostró positividad para c-KIT y CD34 en el 100\% y $63 \%$ de los casos.

PALABRAS CLAVE: GIST; Tumor estromal; Gastrointestinal.

\section{INTRODUCCIÓN}

Los tumores del estroma gastrointestinal (GIST) son neoplasias de origen mesenquimático, que hasta hace 2 décadas eran denominados con diversos nombres de acuerdo a su diferenciación muscular liso (leiomioma, leiomioblastoma o leiomiosarcoma) o neural (schwannoma), permitiendo posteriores estudios de inmunohistoquímica, microscopía electrónica y biología molecular reclasificar a estos tumores como una entidad clínico-patológica propia caracterizada inmunohistoquímicamente y a diferencia de otros tumores de origen muscular liso y neural, por expresar en el $95 \%$ de los casos el receptor de transmembrana para el factor derivado de stem cells con actividad sobre el receptor tirosina-quinasa conocido como CD117 o c-KIT, ubicado en el cromosoma 4q11-q12 y compuesto por 21 exones, siendo las mutaciones a nivel del exón 11 (67\%) y 9 (11\%) las mas frecuentemente detectadas. Otros marcadores frecuentemente positivos pero no específicos incluyen CD34 (60$70 \%$ de los casos) y actina músculo liso (30-40\% de los casos), menos común S-100 (5\%), desmina (raro) y marcadores neuroendocrinos (enolasa neuronal específica y cromogranina) (Rosai, 2004, Rubin et al., 2000; Fletcher et al., 2002; Miettinem et al., 2001; Miettinen et al., 2003).

Los GIST afectan generalmente a sujetos entre la $4^{\circ}$ $6^{\circ}$ décadas de la vida con un leve predominio en población masculina correspondiendo aproximadamente al 0,1-0,3\% 
de todas las neoplasias del tubo digestivo con una incidencia y prevalencia estimada de 14,5 y 129 casos por millón de habitantes respectivamente; representando sin embargo, el 80\% de las neoplasias mesenquimáticas gastrointestinales (Nilsson et al., 2005; Blackstein et al., 2006; Miettinem). Estos tumores se originan a partir de las células intersticiales de Cajal, ubicadas en los plexos mientéricos de la pared gastrointestinal, pudiendo por tanto localizarse en cualquier porción del tubo digestivo, siendo estomago (60-70\%) y duodeno (20-30\%) los más frecuentemente afectados; menos comúnmente colon, esófago y recto, reportándose en forma excepcional compromiso extra-intestinal en omento, mesenterio y retroperitoneo (Rosai; Miettinem et al.; Kindblom et al., 1998; Miettinen et al., 2006).

El recuento mitótico y tamaño tumoral son los principales factores pronósticos identificados relacionados con progresión de la enfermedad y supervivencia, resultando en ocasiones, sin embargo; difícil de predecir su comportamiento biológico (benigno/maligno), razón por la cual las principales clasificaciones vigentes recomiendan más bien catalogarlos de acuerdo al riesgo asociado a metástasis y supervivencia, existiendo actualmente según la clasificación propuesta por la OMS 8 grupos pronósticos (Tabla I), hecho que demuestra el variable comportamiento de esta neoplasia y por lo tanto lo complejo que resulta tratar de definir si estos tumores presentaran un comportamiento benigno, incierto (borderline) o maligno (Fletcher et al.; Bosman et al., 2009; Miettinen et al., 2005; Miettinen et al., 2003; Demetri et al., 2007). Otras variables morfológicas pronósticas consideran el compromiso de la mucosa, presencia de necrosis, hipercelularidad, pleomorfismo celular y localización anatómica.

El objetivo de este estudio fue describir aspectos clínicos, morfológicos y de inmunohistoquímica de pacientes con diagnóstico de GIST realizados en la Unidad de Anatomía Patológica del Hospital Hernán Henríquez Aravena de Temuco, Chile, entre Enero de 1999 y Agosto de 2010.

\section{MATERIAL Y MÉTODO}

\section{Diseño del estudio: Cohorte retrospectiva.}

Participantes: Se incluyeron todos los pacientes con diagnóstico de GIST realizados en la Unidad de Anatomía Patológica del Hospital Hernán Henríquez Aravena de TemucoChile entre Enero de 1999 y Agosto de 2010 (n= 30). Los sujetos incluidos en el estudio fueron confirmados en su diagnóstico mediante la revisión dirigida de las láminas histológicas. Se utilizó un muestreo no probabilístico de casos consecutivos.

Variables de interés: Se estudiaron variables clínicas (edad y género) obtenidas a partir de los registros de las historias clínicas de cada sujeto, morfológicas (localización anatómica, tamaño tumoral, variante histológica, recuento mitótico, compromiso de mucosa, presencia de necrosis, pleomorfismo celular y tipo de cirugía realizada) y de inmunohistoquímica (c-KIT, CD34 y S-100), obtenidas a partir del estudio de las piezas quirúrgicas y láminas histológicas.

Medidas: Algunas de las siguientes variables en estudio fueron categorizadas: Edad, género, tamaño tumoral (medida en $\mathrm{mm}$ ), localización tumoral, tipo histológico (fusocelular, epiteloídeo o mixto), recuento mitótico $(\leq 5$ o $>5$ mitosis por 50 campos de aumento mayor [CAM]), grado de pleomorfismo nuclear (leve, moderado o intenso), compromiso neoplásico de la mucosa, presencia de necrosis ( $\sin$ necrosis, $1-30 \%, 31-50 \%$ y $>50 \%$ ), y número de linfonodos comprometidos por tumor. Cada uno de los tumores fue categorizado según grupo de riesgo o pronóstico en base a la clasificación propuesta por la OMS considerando para ello el tamaño tumoral y recuento mitótico (Tabla I) (Bosman et al.). En relación a la localización tumoral se diferenciaron los tumores de tubo digestivo (esófago, estómago, intestino delgado o grueso) y

Tabla I. Grupos pronósticos según progression de la enfermedad en pacientes con GIST basado en el tamaño tumoral y recuento mitótico (xx).

\begin{tabular}{ccccc}
\hline Grupo pronóstico & Tamaño tumoral & $\begin{array}{c}\text { Recuento mitótico } \\
\left(\mathbf{n}^{\mathbf{0}} \text { mitosis/50 CAM) }\right.\end{array}$ & \multicolumn{2}{c}{ Progresión de la enfermedad (\% de pacientes) } \\
\cline { 4 - 5 } Grupo 1 & $\leq 2 \mathrm{~cm}$. & $\leq 5$ & Est ómago & I. Delgado \\
Grupo 2 & $>2-\leq 5 \mathrm{~cm}$. & $\leq 5$ & 1,9 & 0 \\
Grupo $3 a$ & $>5-\leq 10 \mathrm{~cm}$ & $\leq 5$ & 3,6 & 24 \\
Grupo $3 b$ & $>10 \mathrm{~cm}$. & $\leq 5$ & 12 & 52 \\
Grupo 4 & $\leq 2 \mathrm{~cm}$. & $>5$ & 0 & 50 \\
Grupo 5 & $>2-\leq 5 \mathrm{~cm}$. & $>5$ & 16 & 73 \\
Grupo $6 a$ & $>5-\leq 10 \mathrm{~cm}$. & $>5$ & 55 & 85 \\
Grupo $6 b$ & $>10 \mathrm{~cm}$. & $>5$ & 86 & 90 \\
\hline
\end{tabular}


aquellos con compromiso primario extra-intestinal. La interpretación inmunohistoquímica de c-KIT, CD34 y S-100 se realizó considerando la tinción citoplasmática y/o de membrana de las células tumorales en una escala de 0 a $3+$, siendo 0 negativo, $1+$ positividad débil, $2+$ moderado y $3+$ positividad intensa.

Métodos estadísticos: Se utilizó estadística descriptiva con cálculo de medidas de tendencia central y extrema; y analítica, aplicando análisis divariados: T-student para variables continuas, chi cuadrado de Pearson y exacto de Fisher para variables categóricas; con un intervalo de confianza de $95 \%$ (IC 95\%).

\section{RESULTADOS}

El promedio de edad de la cohorte fue 60 años (17 a 81 años), siendo la distribución según grupos etáreos la siguiente: < 20 años 1, 21-50 años 11, 51-75 años 15 y > 75 años 5 ; sin observarse diferencias estadísticas significativas según localización (Tabla II). Se verificaron en el grupo estudiado $60 \%$ (18) de mujeres y $40 \%$ (12) de hombres.

El 90\% (27) correspondió a tumores de localización gastro-intestinal (1 esófago, 12 estómago, 12 intestino delgado y 2 intestino grueso) (Fig. 1) y $10 \%$ extra-intestinal, todos ellos con compromiso primario de retroperitoneo. El tamaño tumoral promedio de acuerdo a la localización fue $35 \mathrm{~mm}$ en esófago, 50,2 $\pm 30,4 \mathrm{~mm}$ en estómago, 90,6 $\pm 66,7 \mathrm{~mm}$ en intestino delgado, $64 \pm 19,7 \mathrm{~mm}$ en intestino grueso y $117,3 \pm 114,9 \mathrm{~mm}$ en retroperitoneo $(\mathrm{p}=0,46)$ (Tabla II). En los tumores gástricos se efectuó gastrectomía subtotal en 5 casos (42\%), gastrectomía total en $6(50 \%)$ y tumorectomía con márgenes negativos en 1 (8\%). Para tumores de intestino se efectuó resección quirúrgica de acuerdo al segmento comprometido.

De las características histológicas analizadas el $77 \%$ (23) correspondió a tumores con patrón fusocelular, $10 \%$ (3) mixto y $13 \%$ (4) epiteloídeo (Fig. 2A-C); verificándose necrosis en un 37\% (11) de los tumores, correspondiendo $64 \%$ (7) entre $1-30 \%, 27 \%$ (3) entre $31-50 \%$ y $9 \%$ (1) $>50 \%$ de necrosis. El recuento mitótico fue $\leq 5 /$ 50 CAM en 15 casos (50\%). En estómago un 83\% (10) presentó recuento $\leq 5$ mitosis mientras en intestino delgado el 83\% (10) presentó > 5 mitosis/50 CAM; demostrándose compromiso de la mucosa en el 67\% (20) del grupo estudiado (Tabla II).

Según el grupo pronóstico se verificó $7 \%$ grupo 1 , $23 \%$ grupo $2,20 \%$ grupo 3, $0 \%$ grupo $4,10 \%$ grupo 5 y $40 \%$ grupo 6; la distribución por localización se resume en Tabla II. En 8 pacientes (27\%) se realizó linfadenectomía, siendo el promedio de linfonodos resecados 14 (3-26), demostrándose compromiso tumoral en un solo paciente quién presentó metástasis en 3 de 6 linfonodos examinados (Fig. 2D).

Se observó positividad para c-KIT en la totalidad de los casos (30), siendo $1+$ en un 20\% (6), $2+$ en un $70 \%$ (21) y $3+$ en el $10 \%$ (3) de los tumores (Fig. 2E-F). Los resultados del estudio inmunohistoquímico se resumen en la Tabla III.

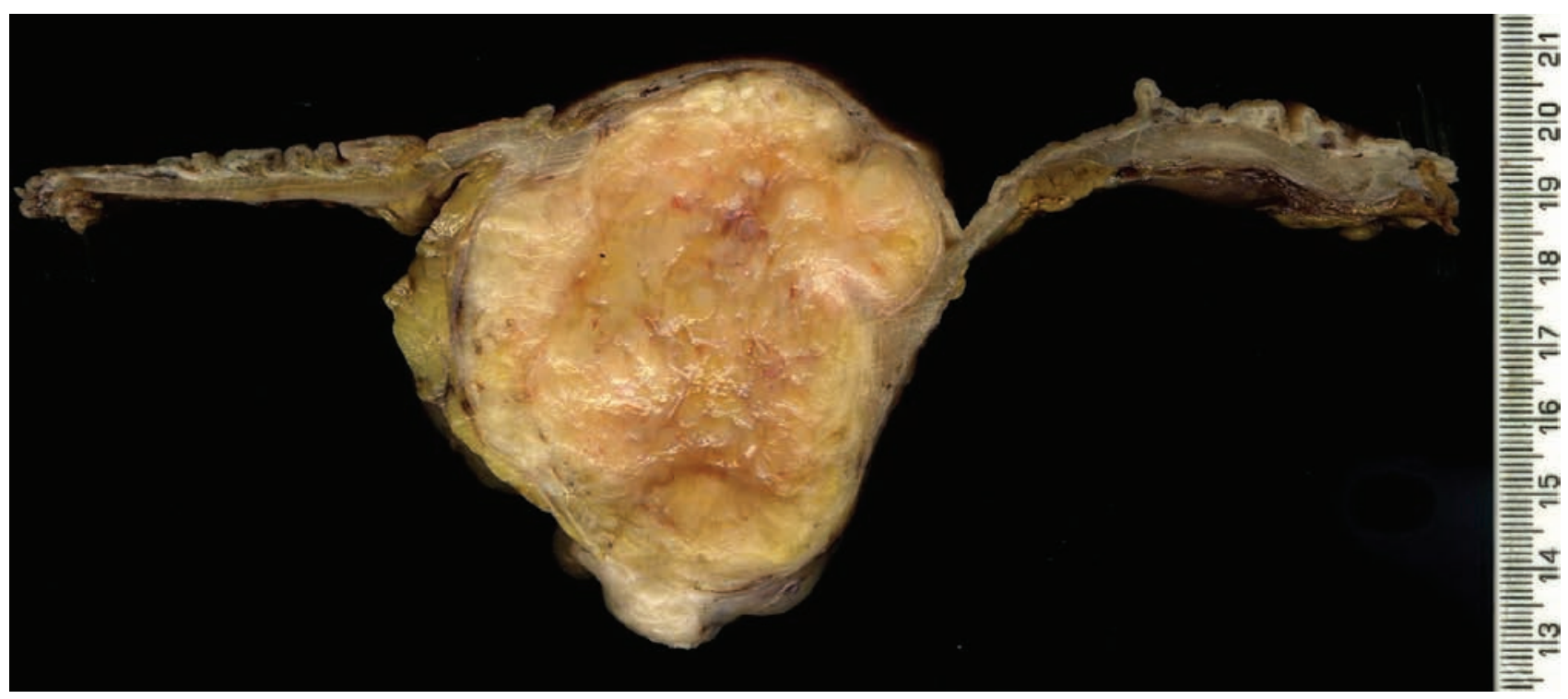

Fig. 1. Pared gástrica con lesión nodular intramural, blanquecino amarillenta, bien delimitada y en partes lobulada, de patrón de crecimiento expansivo que desplaza la mucosa. No se observan áreas de hemorragia ni necrosis. 

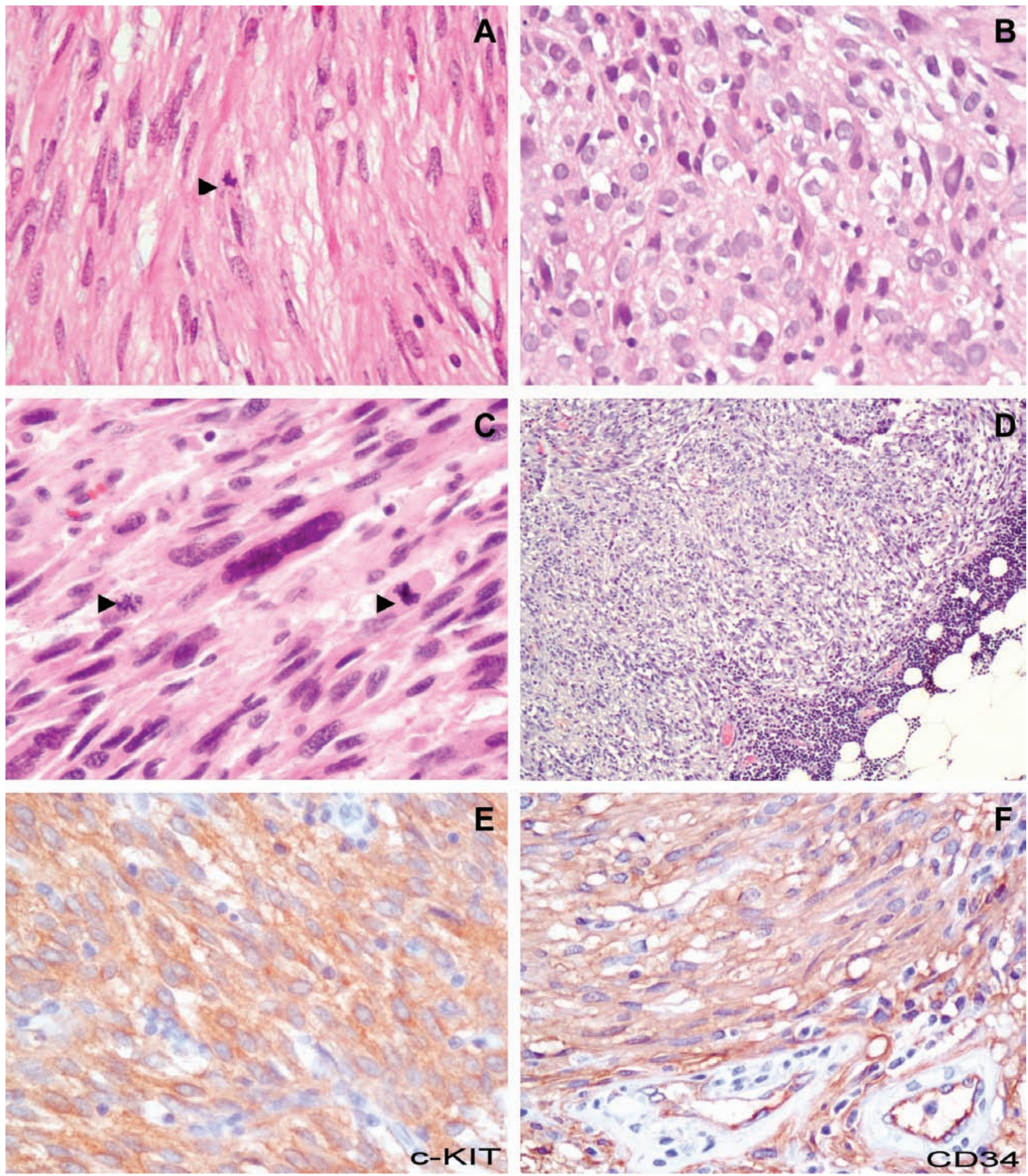

Fig. 2. A (HE, 200x), B (HE, 200x), C (HE, 400x), D (HE, 100x), E-F (200x). En A se aprecia proliferación neoplásica compuesta por células elongadas (patron fusocelular) con pleomorfismo leve y escasas mitosis $(\triangleright)$ ). B, neoplasia estromal compuesta predominantemente por células redondeadas en partes con citoplasma claro de patron epiteloídeo. C, tumor estromal fusocelular con acentuado pleomorfismo nuclear y abundantes mitosis $(\bullet)$. D, metastasis ganglionar linfática de GIST. E y F, células tumorales con positividad citoplasmática intensa (3+) y difusa para c-KIT y CD34. 
TAPIA, E. O. \& ROA, S. J. C. Tumores del estroma gastrointestinal (GIST): características clinico-morfológicas y perfil inmunohistoquímico. Int. J. Morphol., 29(I):244-251, 2011.

Tabla II. Distribución de variables clínicas y morfológicas según localización tumoral.

\begin{tabular}{|c|c|c|c|c|c|}
\hline \multirow{2}{*}{ Variable } & Esófago & Estómago & I. Delgado & I. Grueso & Extra-intestinal \\
\hline & $3 \%(1)$ & $40 \%(12)$ & $40 \%(12)$ & $7 \%(2)$ & $10 \%(3)$ \\
\hline Edad (promedio) & 62 años & $55,9 \pm 18,4$ años & $62,5 \pm 13,3$ años & $75 \pm 8,4$ años & $56 \pm 15,8$ años \\
\hline \multicolumn{6}{|l|}{ Género } \\
\hline Femenino & $0 \%(0)$ & $58 \%(7)$ & $58 \%(7)$ & $100 \%(2)$ & $67 \%(2)$ \\
\hline Masculino & $100 \%(1)$ & $42 \%(5)$ & $42 \%(5)$ & $0 \%(0)$ & $33 \%(1)$ \\
\hline Tamaño tumoral (promedio) & $35 \mathrm{~mm}$. & $50,2 \pm 30,4 \mathrm{~mm}$ & $90,6 \pm 66,7 \mathrm{~mm}$ & $64 \pm 19,7 \mathrm{~mm}$ & $117,3 \pm 114,9 \mathrm{~mm}$ \\
\hline \multicolumn{6}{|l|}{ Tamaño tumoral } \\
\hline$\leq 2 \mathrm{~cm}$ & $0 \%(0)$ & $8 \%(1)$ & $8 \%(1)$ & $0 \%(0)$ & $0 \%(0)$ \\
\hline$>2-\leq 5 \mathrm{~cm}$ & $100 \%(1)$ & $50 \%(6)$ & $8 \%(1)$ & $50 \%(1)$ & $33 \%(1)$ \\
\hline$>5-\leq 10 \mathrm{~cm}$ & $0 \%(0)$ & $42 \%(5)$ & $50 \%(6)$ & $50 \%(1)$ & $33 \%(1)$ \\
\hline$>10 \mathrm{~cm}$ & $0 \%(0)$ & $0 \%(0)$ & $34 \%(4)$ & $0 \%(0)$ & $33 \%(1)$ \\
\hline \multicolumn{6}{|l|}{ Pleomorfismo celular } \\
\hline Leve & $100 \%(1)$ & $67 \%(8)$ & $67 \%(8)$ & $0 \%(0)$ & $67 \%(2)$ \\
\hline Moderado & $0 \%(0)$ & $25 \%(3)$ & $8 \%(1)$ & $100 \%(2)$ & $33 \%(1)$ \\
\hline Intenso & $0 \%(0)$ & $8 \%(1)$ & $25 \%(3)$ & $0 \%(0)$ & $0 \%(0)$ \\
\hline \multicolumn{6}{|l|}{ Patrón Histológico } \\
\hline Fusocelular & $0 \%(0)$ & $75 \%(9)$ & $75 \%(9)$ & $100 \%(2)$ & $100 \%$ \\
\hline Epiteloídeo & $100 \%(1)$ & $17 \%(2)$ & $8 \%(1)$ & $0 \%(0)$ & $0 \%(0)$ \\
\hline Mixto & $0 \%(0)$ & $8 \%(1)$ & $17 \%(2)$ & $0 \%(0)$ & $0 \%(0)$ \\
\hline \multicolumn{6}{|l|}{ Presencia Necrosis } \\
\hline Ausente & $100 \%(1)$ & $83 \%(10)$ & $25 \%(3)$ & $100 \%(2)$ & $100 \%(3)$ \\
\hline $1-30 \%$ & $0 \%(0)$ & $17 \%(2)$ & $42 \%(5)$ & $0 \%(0)$ & $0 \%(0)$ \\
\hline $30-50 \%$ & $0 \%(0)$ & $0 \%(0)$ & $25 \%(3)$ & $0 \%(0)$ & $0 \%(0)$ \\
\hline$>50 \%$ & $0 \%(0)$ & $0 \%(0)$ & $8 \%(1)$ & $0 \%(0)$ & $0 \%(0)$ \\
\hline \multicolumn{6}{|l|}{ Recuento mitótico* } \\
\hline$\leq 5$ mitosis & $100 \%(1)$ & $83 \%(10)$ & $17 \%(2)$ & $0 \%(0)$ & $67 \%(2)$ \\
\hline$>5$ mitosis & $0 \%(0)$ & $17 \%(2)$ & $83 \%(10)$ & $100 \%(2)$ & $33 \%(1)$ \\
\hline \multicolumn{6}{|l|}{ Grupo pronóstico } \\
\hline Grupo 1 & $0 \%(0)$ & $8 \%(1)$ & $8 \%(1)$ & $0 \%(0)$ & $0 \%(0)$ \\
\hline Grupo 2 & $100 \%(1)$ & $50 \%(6)$ & $0 \%(0)$ & $0 \%(0)$ & $0 \%(0)$ \\
\hline Grupo 3 & $0 \%(0)$ & $25 \%(3)$ & $8 \%(1)$ & $0 \%(0)$ & $67 \%(2)$ \\
\hline Grupo 4 & $0 \%(0)$ & $0 \%(0)$ & $0 \%()$ & $0 \%(0)$ & $0 \%(0)$ \\
\hline Grupo 5 & $0 \%(0)$ & $0 \%(0)$ & $8 \%(1)$ & $50 \%(1)$ & $33 \%$ (1) \\
\hline Grupo 6 & $0 \%(0)$ & $17 \%(2)$ & $75 \%(9)$ & $50 \%(1)$ & $0 \%(0)$ \\
\hline
\end{tabular}

\section{DISCUSIÓN}

Los tumores del estroma gastrointestinal (GIST) son neoplasias de origen mesenquimático, constituyendo un grupo de lesiones que inicialmente fueron denominados de acuerdo a su diferenciación muscular liso o neural como leiomioma, leiomioblastomas, leiomiosarcomas o schwannomas; siendo en 1983 introducido el término GIST 
TAPIA, E. O. \& ROA, S. J. C. Tumores del estroma gastrointestinal (GIST): características clinico-morfológicas y perfil inmunohistoquímico. Int. J. Morphol., 29(I):244-251, 2011.

Tabla III. Perfil inmunohistoquímico según localización tumoral.

\begin{tabular}{|c|c|c|c|c|c|c|}
\hline Anticuerpo & $\begin{array}{c}\text { Esófago } \\
3 \%(1)\end{array}$ & $\begin{array}{c}\text { Estómago } \\
40 \%(12)\end{array}$ & $\begin{array}{c}\text { I. Delgado } \\
40 \%(12)\end{array}$ & $\begin{array}{c}\text { I. Grueso } \\
7 \%(2)\end{array}$ & $\begin{array}{c}\text { Extra-intestinal } \\
10 \%(3)\end{array}$ & $\begin{array}{c}\text { Total } \\
100 \%(30)\end{array}$ \\
\hline \multicolumn{7}{|l|}{$c-K I T$} \\
\hline Positivo & $100 \%(1)$ & $100 \%(12)$ & $100 \%(12)$ & $100 \%(2)$ & $100 \%$ & $100 \%(30)$ \\
\hline $1+$ & $0 \%(0)$ & $8 \%(1)$ & $0 \%(0)$ & $0 \%(0)$ & $100 \%(3)$ & $13 \%(4)$ \\
\hline $2+$ & $100 \%$ & $75 \%(9)$ & $17 \%(2)$ & $100 \%$ & $0 \%(0)$ & $47 \%(14)$ \\
\hline $3+$ & $0 \%(0)$ & $17 \%(2)$ & $83 \%(10)$ & $0 \%(0)$ & $0 \%(0)$ & $40 \%(12)$ \\
\hline Negativo & $0 \%(0)$ & $0 \%(0)$ & $0 \%(0)$ & $0 \%(0)$ & $0 \%(0)$ & $0 \%(0)$ \\
\hline \multicolumn{7}{|l|}{ CD34 } \\
\hline Positivo & $0 \%(0)$ & $75 \%(9)$ & $67 \%(8)$ & $0 \%(0)$ & $67 \%(2)$ & $63 \%$ (19) \\
\hline $1+$ & $0 \%(0)$ & $33 \%(3)$ & $0 \%(0)$ & $0 \%(0)$ & $50 \%(1)$ & $21 \%(4)$ \\
\hline $2+$ & $0 \%(0)$ & $56 \%(5)$ & $75 \%(6)$ & $0 \%(0)$ & $50 \%(1)$ & $63 \%(12)$ \\
\hline $3+$ & $0 \%(0)$ & $11 \%(1)$ & $25 \%(2)$ & $0 \%(0)$ & $0 \%(0)$ & $16 \%(3)$ \\
\hline Negativo & $100 \%(1)$ & $25 \%(3)$ & $33 \%(4)$ & $100 \%(2)$ & $33 \%(1)$ & $37 \%(11)$ \\
\hline \multicolumn{7}{|l|}{$S-100$} \\
\hline Positivo & $0 \%(0)$ & $8 \%(1)$ & $0 \%()$ & $0 \%(0)$ & $0 \%(0)$ & $3 \%(1)$ \\
\hline $1+$ & $0 \%(0)$ & $100 \%$ & $0 \%(0)$ & $0 \%(0)$ & $0 \%(0)$ & $100 \%$ \\
\hline $2+$ & $0 \%(0)$ & $0 \%(0)$ & $0 \%(0)$ & $0 \%(0)$ & $0 \%(0)$ & $0 \%(0)$ \\
\hline $3+$ & $0 \%(0)$ & $0 \%(0)$ & $0 \%(0)$ & $0 \%(0)$ & $0 \%(0)$ & $0 \%(0)$ \\
\hline Negativo & $100 \%$ & $92 \%(11)$ & $100 \%(12)$ & $100 \%(2)$ & $100 \%(3)$ & $97 \%(29)$ \\
\hline
\end{tabular}

por Mazur \& Clark para referirse a a una entidad clínicopatológica caracterizada inmunohistoquímicamente por expresar en más del $90 \%$ de los casos el receptor de transmembrana para el factor derivado de stem cells con actividad sobre el receptor tirosina-quinasa conocido como CD117 o c-KIT (Rosai; Rubin et al.; Fletcher et al.; Miettinem et al.; Mazur \& Clark, 1983).

Se trata de tumores infrecuentes que representan no mas allá del 0,3\% de todas las neoplasias gastro-intestinales, siendo sin embargo, la neoplasia no epitelial más frecuente del tubo digestivo. Dado su origen en las células intersticiales de Cajal pueden afectar cualquier segmento del tracto gastro-intestinal, verificando en nuestra serie al igual que otros autores que estómago e intestino delgado son los órganos más frecuentemente comprometidos, representando en conjunto el $80 \%$ de los casos. Aún cuando trabajos mencionan como excepcional el compromiso extraintestinal, en 3 pacientes (10\%) el compromiso primario fue retroperitoneal. El 67\% de los casos correspondió a pacientes mayores de 50 años, siendo por tanto la $4^{\circ}-6^{\circ}$ décadas de la vida el grupo etáreo mayormente afectado. (Nilsson et al.; Blackstein et al.; Miettinem et al.; Rosai; Kindblom et $a l$. .). Si bien la mayor parte de los trabajos publicados no reportan diferencias por género o un leve predominio en hombres, en nuestro grupo el $60 \%$ correspondió a mujeres, al igual que lo reportado por Mucciarini et al. (2007) con un ligero predominio (53\% mujeres).

El patrón fusocelular es el más frecuentemente observado, representando en nuestro trabajo el $77 \%$, seguido del epiteloídeo (13\%) y mixto (10\%).

El comportamiento biológico impredecible y potencialmente agresivo que presentan estos tumores dificulta catalogarlas como benignas, limítrofes o malignas; siendo el recuento mitótico y tamaño tumoral los principales factores morfológicos pronósticos relacionados con progresión de la enfermedad y supervivencia en este grupo de pacientes. Lo anteriormente mencionado explica que se recomiende hablar más bien de grupo pronóstico o de riesgo en lugar de lesiones benignas o malignas, siendo el tamaño e índice mitótico la base de estas clasificaciones, utilizando en nuestro estudio la última clasificación propuesta por la OMS en diciembre de 2009 (Bosman et al.) (Tabla I). De acuerdo a ella, el 50\% correspondió a grupo 5-6, por lo tanto con recuento mitótico > 5/50 CAM. Otros elementos pronósticos incluyen la presencia de necrosis y compromiso de la mucosa, observándose necrosis en $1 / 3$ de los casos mientras que el compromiso de la mucosa alcanzó el 67\%. 
En el estudio inmunohistoquímico, los GIST se caracterizan por expresar c-KIT con tan solo un pequeño porcentaje $(<5 \%)$ que son negativos, verificando en nuestra serie que la totalidad de casos presentaron inmunopositividad para C-KIT, sin embargo, se debe tener presente que la negatividad de este marcador en presencia de un patrón histológico sugerente no descarta el diagnóstico (Miettinen et al.; Rubin et al.; Fletcher et al.). Otros marcadores útiles pero menos específicos consideran el CD34 y S-100, reportándose positividad entre $60-70 \%$ y $5 \%$ para cada uno respectivamente; siendo en nuestro grupo de estudio $63 \%$ y $3 \%$.

El tratamiento de esta neoplasia es quirúrgico y consiste idealmente en la resección completa con márgenes ne- gativos (R0), dependiendo esta de la localización, tamaño y compromiso de estructuras vecinas, no recomendándose la disección de los linfonodos, dado lo infrecuente de su compromiso y diseminación más frecuentemente por vía hematógena con compromiso hepático (DeMatteo et al., 2000; Patel et al., 2000; Corless et al., 2002; Crosby et al., 2001; Fletcher et al.; Miettinen et al). En nuestro trabajo, en 8 casos se realizó disección de linfonodos identificándose en 1 de ellos 3 metástasis (Fig. 2D) (localización: intestino delgado, tamaño: $85 \mathrm{~mm}, 26$ mitosis/50 CAM, grupo pronóstico 6A); lo que plantea la necesidad de evaluar en grupos de alto riesgo ( 5 y 6 ) el beneficio de efectuar la linfadenectomía y de esta forma asegurar en estos pacientes una cirugía con intención curativa.

TAPIA, E. O. \& ROA, S. J. C. Gastrointestinal stromal tumors (GIST): clinical-morphological features and immunohistochemical profile. Int. J. Morphol., 29(1):244-251, 2011.

SUMMARY: Gastrointestinal stromal tumors (GIST) are mesenchymal neoplasms that represent approximately $0.3 \%$ of all malignancies of the gastrointestinal tract, characterized immunohistochemically for CD117 expression in 95\% of cases and most commonly affects the stomach and small intestine. The aim of this study is to describe the clinical, morphological and immunohistochemical diagnosis of GIST patients in the unit of pathology Hernán Henríquez Aravena Hospital in Temuco. Retrospective cohort study. We studied 30 patients with gist who underwent surgery between 1999 and 2010 in the Hernan Henríquez Aravena Hospital in Temuco. The clinical and morphological variables studied were age, gender, location and tumor size, histological type, mitotic index, commitment mucosa, degree of nuclear pleomorphism and necrosis. immunohistochemical study found c-KIT, CD34 and S-100. Descriptive statistics and analytical, using Pearson chi-square and Fisher exact tests for categorical variables, and T-test for continuous variables. The average age was 60 years (1781 years), verified $60 \%$ of women in the study group. $90 \%$ corresponded to tumors located gastro-intestinal, stomach and small intestine represents $80 \%$ of cases. The average tumor size was $75.9 \mathrm{~mm}$. spindle pattern accounted for $77 \%$, with necrosis in $37 \%$ of cases. $50 \%$ had $>$ 5 mitosis $/ 50$ cam, mucosal involvement verified by $67 \%$. according to the prognostic group was observed $7 \%$ group 1 , group $223 \%, 20 \%$ in group 3, $0 \%$ in group 4, $10 \%$ and $40 \%$ group 5 group $6.100 \%$ expressed positive for c-KIT, CD34 63\% and 3\% for S-100. GIST mostly affect patients from the 4 th -6 decade of life with a slight female predominance, stomach and small intestine being the organs most commonly affected. Immunohistochemical study showed positivity for c-KIT and CD34 in 100\% and 63\% of cases.

KEY WORDS: GIST; Stromal tumor; Gastrointestinal.

\section{REFERENCIAS BIBLIOGRÁFICAS}

Bosman, F.; Carneiro, F.; Hruban, R. \& Theise, N. WHO classification of tumours of the digestive system. $4^{\text {th }} \mathrm{ed}$. International Agency for Research Cancer-IARC, 2009.

Corless, C.; McGreevey, L.; Haley, A.; Town, A. \& Heinrich, M. KIT mutations are common in incidental gastrointestinal stromal tumors one centimetre or less in size. Am. J. Pathol., 160:1567-72, 2002.

Crosby, J. A.; Catton, C. N., Davis, A.; Couture, J.; O'Sullivan, B.; Kandel, R. \& Swallow, C. J. Malignant gastrointestinal stromal tumors of the small intestine: a review of 50 cases from a prospective database. Ann. Surg. Oncol., 8:50-9, 2001.
DeMatteo, R.; Lewis, L.; Leung, D.; Mudan, S.; Woodruff, J. \& Brennan, M. Two hundred gastrointestinal stromal tumors. Recurrence patterns and prognostic factors for survival. Ann. Surg., 231:51-8, 2000.

Demetri, G. D.; Benjamin, R. S.; Blanke, C. D.; Blay, J. Y.; Casali, P.; Choi, H.; Corless, C. L.; Debiec-Rychter, M.; DeMatteo, R. P.; Ettinger, D. S.; Fisher, G. A.; Fletcher, C. D.; Gronchi, A.; Hohenberger, P.; Hughes, M.; Joensuu, H.; Judson, I.; Le Cesne, A.; Maki, R. G.; Morse, M.; Pappo, A. S.; Pisters, P. W.; Raut, C. P.; Reichardt, P.; Tyler, D. S.; Van den, Abbeele, A. D.; von Mehren, M.; Wayne, J. D.; Zalcberg, J. \& NCCN Task Force. NCCN Task Force report: management of patients 
with gastrointestinal stromal tumor (GIST)--update of the NCCN clinical practice guidelines. J. Natl. Compr. Canc. Netw., 5:S1-S29, 2007.

Fletcher, C. D.; Berman, J. J.; Corless, C.; Gorstein, F.; Lasota, J.; Longley, B. J.; Miettinen, M.; O'Leary, T. J.; Remotti, H.; Rubin, B. P.; Shmookler, B.; Sobin, L. H. \& Weiss, S. W. Diagnosis of gastrointestinal stromal tumors: A consensus approach. Hum. Pathol., 33:459-65, 2002.

Kindblom, L. G.; Remotti, H. E.; Aldenborg, F. \& MeisKindblom, J. M: Gastrointestinal pacemaker cell tumor (GIPACT): gastrointestinal stromal tumors show phenotypic characteristics of the interstitial cells of Cajal. Am. J. Pathol., 152:1259-69, 1998.

Mazur, M. T. \& Clark, H. B. Gastric stromal tumors. Reappraisal of histogenesis. Am. J. Surg. Pathol., 7: 50719, 1983.

Blackstein, M. E.; Blay, J. Y.; Corless, C.; Driman, D. K.; Riddell, R.; Soulières, D.; Swallow, C. J.; Verma, S. \& Canadian Advisory Committee on GIST. Gastrointestinal stromal tumours: Consensus statement on diagnosis and treatment. Can. J. Gastroenterol., 20:157-63, 2006.

Miettinem, M. \& Lasota, J. Gastrointestinal stromal tumors (GISTs): definition, ocurrence, pathology, differential diagnosis, and molecular genetics. Pol. J. Pathol., 54:324, 2003.

Miettinen, M.; Furlong. M.; Sarlomo-Rikala, M.; Burke, A.; Sobin, L. H. \& Lasota, J. Gastrointestinal stromal tumors, intramural leiomyomas, and leiomyosarcomas in the rectum and anus: a clinicopathologic, immunohistochemical, and molecular genetic study of 144 cases. Am. J. Surg. Pathol., 25:1121-33, 2001.

Miettinen, M.; Kopczynski, J.; Makhlouf, H. R.; SarlomoRikala, M.; Gyorffy, H.; Burke, A.; Sobin, L. H. \& Lasota, J. Gastrointestinal stromal tumors, intramural leiomyomas, and leiomyosarcomas in the duodenum: a clinicopathologic,immunohistochemical, and molecular genetic study of 167 cases. Am. J. Surg. Pathol., 27: 62541, 2003.

Miettinen, M. \& Lasota, J. Gastrointestinal stromal tumorsdefinition, clinical, histological, immunohistochemical, and molecular genetic features and differential diagnosis. Virchows Arch., 438:1-12, 2001.

Miettinen, M. \& Lasota, J. Gastrointestinal stromal tumors: pathology and prognosis at different sites. Semin. Diagn. Pathol., 23:70-83, 2006.
Miettinen, M. \& Lasota, J. Gastrointestinal stromal tumors: review on morphology, molecular pathology, prognosis, and differential diagnosis. Arch. Pathol. Lab. Med., 130:1466-78, 2006.

Miettinen, M.; Makhlouf, H.; Sobin, L. H. \& Lasota, J. Gastrointestinal stromal tumors of the jejunum and ileum: a clinicopathologic, immunohistochemical, and molecular genetic study of 906 cases before imatinib with long-term follow-up. Am. J. Surg. Pathol., 30: 47789, 2006.

Miettinen, M.; Sobin, L. H. \& Lasota, J. Gastrointestinal stromal tumors of the stomach: a clinicopathologic, immunohistochemical, and molecular genetic study of 1765 cases with long-term follow-up. Am. J. Surg. Pathol., 29:52-68, 2005.

Mucciarini, C.; Rossi, G.; Bertolini, F.; Valli, R.; Cirilli, C.; Rashid, I.; Marcheselli, L.; Luppi, G. \& Federico, M. Incidence and clinicopathologic features of gastrointestinal stromal tumors. A population-based study. BMC Cancer, 7: 230-7, 2007.

Nilsson, B.; Bümming, P.; Meis-Kindblom, J. M.; Odén, A.; Dortok, A.; Gustavsson, B.; Sablinska, K. \& Kindblom, L. G. Gastrointestinal stromal tumors: The incidence, prevalence, clinical course, and prognostication in the preimatinib mesylate era--a population-based study in western Sweden. Cancer, 103:821-9, 2005.

Patel, S. R. \& Benajamin, R. S. Management of peritoneal and hepatic metastases from gastrointestinal stromal tumours. Surg. Oncol., 9:67-70, 2000.

Rosai, J. Stromal tumors. In: Rosai \& Ackerman's Surgical Pathology. $9^{\text {th }}$ Ed. St. Louis, Mosby, 2004. pp.674-80. Vol 1, Chapter 11.

Rubin, B.; Fletcher, J. \& Fletcher, C. Molecular insights into the histogenesis and pathogenesis of gastrointestinal stromal tumors. Int. J. Surg. Pathol., 8:5-10, 2000.

\section{Dirección para correspondencia:}

Dr. Oscar Tapia E.

Departamento de Anatomía Patológica,

Facultad de Medicina

Universidad de La Frontera.

Manuel Montt 112.

Código Postal 478-1176.

Temuco,

Recibido : 22-11-2010

CHILE

Aceptado: 15-12-2010

E-mail: otescalona@gmail.com 